\title{
Contents of Volume II
}

10. Quantum Gases Without Interactions ............ 1

10.1 The Indistinguishability of Quantum Particles $\ldots \ldots \ldots \ldots 3$

10.1.1 Exchange of Two Identical Particles ......... 3

10.1.2 The Pauli Principle $\ldots \ldots \ldots \ldots \ldots \ldots \ldots \ldots \ldots$

10.1.3 Bosons and Fermions;

Elementary and Composite Particles ........ 8

10.1.4 Effects Connected with Indistinguishability $\ldots . \ldots 11$

10.2 Fock Bases . . . . . . . . . . . . . . . . . 14

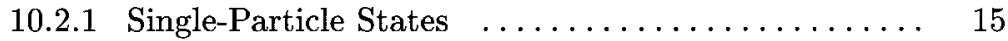

10.2 .2 Occupation Numbers $\ldots \ldots \ldots \ldots \ldots \ldots \ldots \ldots \ldots \ldots \ldots$

10.2.3 Operators in the Fock Representation ........ 20

10.3 Equilibrium of Quantum Gases $\ldots \ldots \ldots \ldots \ldots \ldots \ldots \ldots \ldots$

10.3.1 Grand Canonical Partition Function $\ldots \ldots \ldots \ldots 24$

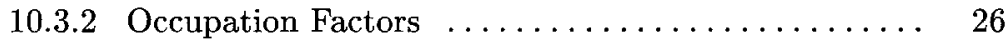

10.3.3 Large Volume Limit; Density of States . . . . . . 27

10.3.4 Classical Limit; Low Densities ............ 31

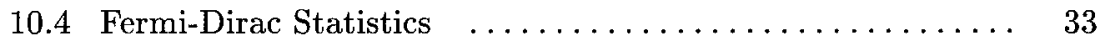

10.4.1 Examples of Fermion Gases . . . . . . . . . 33

10.4.2 Fermion Gas at Zero Temperature;

Fermi Temperature . . . . . . . 35

10.4.3 Equilibrium Properties at Low Temperatures .... 37

10.5 Bose-Einstein Statistics $\ldots \ldots \ldots \ldots \ldots \ldots \ldots \ldots \ldots \ldots \ldots \ldots$

10.5.1 Examples of Boson Gases $\ldots \ldots \ldots \ldots \ldots \ldots \ldots \ldots 43$

10.5.2 Chemical Potentials of Boson Gases $\ldots \ldots \ldots \ldots 44$

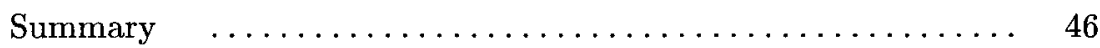

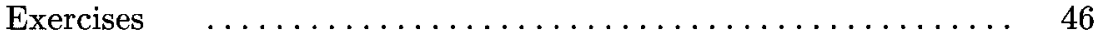

10a Indistinguishable Particles in a Harmonic Potential $\ldots \ldots \ldots \ldots \ldots \ldots \ldots \ldots$

10b Pauli Paramagnetism $\ldots \ldots \ldots \ldots \ldots \ldots \ldots \ldots \ldots \ldots$

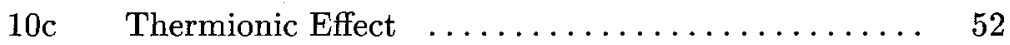

10d Metallic Film .................... 53

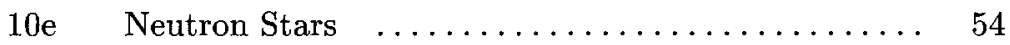

$10 \mathrm{f}$ Quantum Corrections to the Perfect Gas ...... 56

$10 \mathrm{~g}$ Spatial Correlations Due to Indistinguishability ... 56 
11. Elements of Solid State Theory $\ldots \ldots \ldots \ldots \ldots \ldots \ldots \ldots, 59$

11.1 Crystal Order $\ldots \ldots \ldots \ldots \ldots \ldots \ldots \ldots \ldots \ldots \ldots, \quad 62$

11.1.1 The Born-Oppenheimer Method ............ 62

11.1.2 Properties Related to the Crystal Structure ...... 65

11.2 Single-Electron Levels in a Periodic Potential . . . . . . . 68

11.2.1 Independent Electron Approximation $\ldots \ldots \ldots \ldots 68$

11.2.2 Introduction to Band Theory $\ldots \ldots \ldots \ldots \ldots \ldots .73$

11.2.3 Weak Binding Limit $\ldots \ldots \ldots \ldots \ldots \ldots \ldots \ldots \ldots \ldots$

11.2.4 Tight Binding Limit $\ldots \ldots \ldots \ldots \ldots \ldots \ldots \ldots, 83$

11.2.5 Three-Dimensional Bands $\ldots \ldots \ldots \ldots \ldots \ldots \ldots .66$

11.3 Electrons in Metals, Insulators, and Semiconductors ..... 90

11.3.1 Metals .......................... 91

11.3.2 Insulators; Dynamics of Electrons and Holes $\ldots . . \quad 95$

11.3.3 Microscopic Foundations of Electrostatic Equilibrium $\ldots \ldots \ldots \ldots \ldots \ldots, 107$

11.3.4 Semiconductors $\ldots \ldots \ldots \ldots \ldots \ldots \ldots \ldots \ldots . \ldots \ldots$

11.3.5 p-n Junctions, Photocell, Diode, and Transistor . . 122

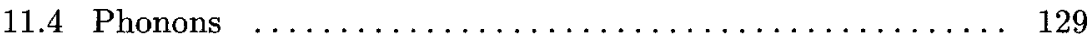

11.4.1 Lattice Vibrations ................... 130

11.4.2 Interpretation of a Mode as a Boson State ...... 136

11.4.3 Specific Heats of Solids ................. 139

11.4.4 Thermal Equilibrium of a Vibrating String $\ldots \ldots .143$

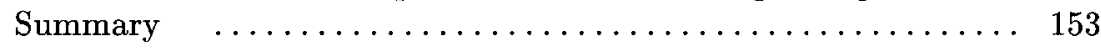

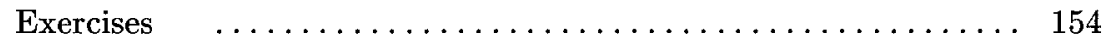

11a Point Defects in Crystals ................. 154

11b Semiconductors in the Extrinsic and Saturation Regimes $\ldots \ldots \ldots \ldots \ldots \ldots \ldots, 155$

11c p-n Junction at Zero Temperature ........... 156

11d Einstein Model ........................ 158

11e Acoustic and Optical Phonons . ............ 158

11f Ferromagnetism of Metals .............. 159

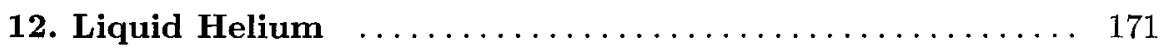

12.1 Peculiar Properties of Helium $\ldots \ldots \ldots \ldots \ldots \ldots \ldots \ldots \ldots \ldots \ldots \ldots$

12.1.1 Phase Diagrams . . . . . . . . . . . . . . . 172

12.1.2 Quantum Liquids $\ldots \ldots \ldots \ldots \ldots \ldots \ldots \ldots \ldots$

12.2 Helium Three ............................ 175

12.2.1 Equation of State $\ldots \ldots \ldots \ldots \ldots \ldots \ldots \ldots \ldots \ldots \ldots \ldots$

12.2.2 Thermal Properties .................. 177

12.2.3 Solid-Liquid Transition . ................ 178

12.3 Helium Four and Bose Condensation $\ldots \ldots \ldots \ldots \ldots \ldots$

12.3.1 Bose Condensation ................... 179

12.3.2 Bose-Einstein Phase Transition $\ldots \ldots \ldots \ldots \ldots \ldots 183$

12.3.3 Superfluidity and Superconductivity $\ldots \ldots \ldots \ldots 186$

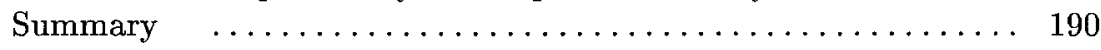


Exercises

12a Specific Heat of the Bose Gas . . . . . . . . . 191

12b Critical Fluctuations ................. 192

12c Bose Condensation in a Canonical Ensemble ..... 192

12d Rôle of the Interactions in the Bose Transition . . . 195

13. Equilibrium and Transport of Radiation $\ldots \ldots \ldots \ldots \ldots 201$

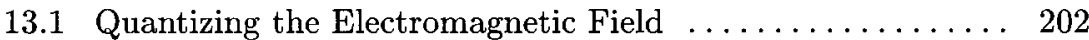

13.1.1 Classical Modes in a Cavity ............. 202

13.1.2 Quantized Radiation Spectrum ............ 205

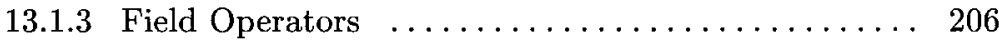

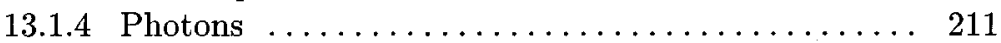

13.2 Equilibrium of Radiation in an Enclosure ......... 215

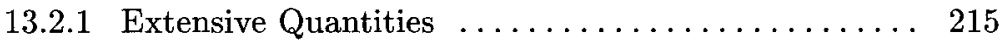

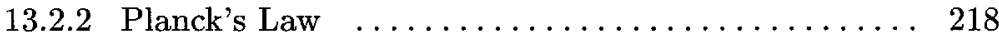

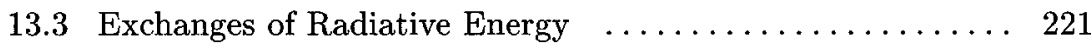

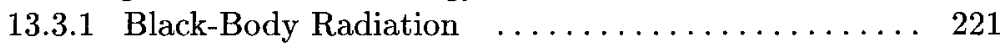

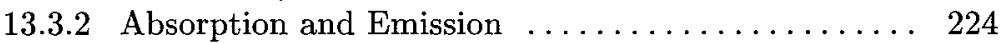

13.3.3 Kirchhoff's Law .................... 227

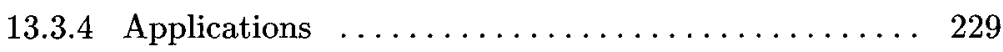

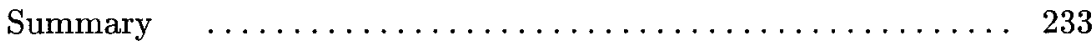

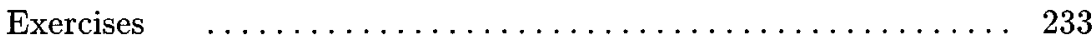

13a Pressure and Internal Energy $\ldots \ldots \ldots \ldots \ldots \ldots 233$

13b Density of Photons in the 3K Radiation ....... 235

13c Neutrinos in Cosmology . . . . . . . . . . . 235

13d Planetary Temperatures .............. 237

$13 \mathrm{e} \quad$ Dewar Flasks ...................... 238

$13 \mathrm{f}$ Greenhouse Effect $\ldots \ldots \ldots \ldots \ldots \ldots \ldots \ldots \ldots \ldots$

14. Non-Equilibrium Thermodynamics $\ldots \ldots \ldots \ldots \ldots \ldots \ldots \ldots 241$

14.1 Conservation Laws $\ldots \ldots \ldots \ldots \ldots \ldots \ldots \ldots \ldots \ldots \ldots \ldots \ldots$

14.1.1 The Problematics of Thermodynamics . . . . . . 243

14.1.2 Different Kinds of Processes . . . . . . . . . . 245

14.1.3 Relations Between Fluxes, Currents, and Densities 249

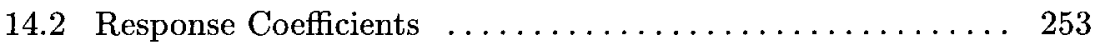

14.2.1 Local Equations of State $\ldots \ldots \ldots \ldots \ldots \ldots \ldots \ldots 25$

14.2.2 Responses of the Fluxes to the Affinities . . . . . . 255

14.2.3 Some Common Transport Coefficients . . . . . . . . 259

14.2.4 Curie's Principle and Onsager's Relations ...... 261

14.2 .5 Dissipation ................... 265

14.2.6 Summary: Macroscopic Approach

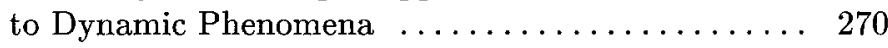

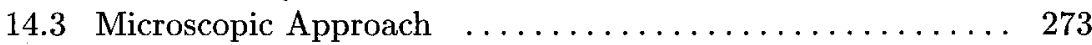

14.3.1 Invariance and Conservation $\ldots \ldots \ldots \ldots \ldots \ldots \ldots \ldots \ldots$

14.3.2 Microscopic Expression for the Fluxes in a Fluid . . 276 
14.3.3 Relevant Entropy $\ldots \ldots \ldots \ldots \ldots \ldots \ldots \ldots \ldots 282$

14.3.4 Macroscopic, Microscopic and Mesoscopic Descriptions ............. 284

14.3.5 The Projection Method $\ldots \ldots \ldots \ldots \ldots \ldots \ldots . \ldots \ldots$

14.4 Applications ....................... 290

14.4.1 Particle and Heat Diffusion $\ldots \ldots \ldots \ldots \ldots \ldots \ldots 291$

14.4 .2 Electrodynamics . . . . . . . . . . . . . 293

14.4.3 Thermoelectric Effects .............. 297

14.4.4 Local Equilibrium of a Fluid ............ 300

14.4.5 Conservation Laws in a Fluid $\ldots \ldots \ldots \ldots \ldots \ldots . \ldots 304$

14.4.6 Equations of Hydrodynamics $\ldots \ldots \ldots \ldots \ldots \ldots . \ldots 308$

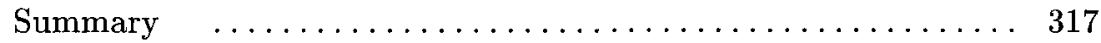

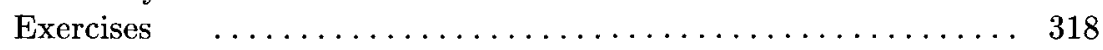

14a Design of an Isotope Separation Plant $\ldots \ldots \ldots .318$

14b Response to an External Perturbation ........ 321

14c Desorption ................... 330

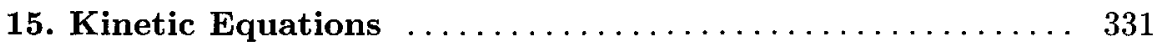

15.1 The Lorentz Model . ..................... 333

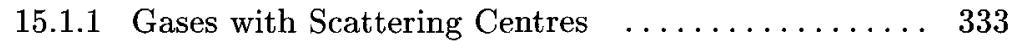

15.1.2 Successive Contractions of the Description ...... . 334

15.1.3 Evolution of the Single-Particle Reduced Density . . 336

15.1.4 Ballistic and Local Equilibrium Regimes . . . . . . 340

15.1.5 The Chapman-Enskog Method ........... 344

15.2 Applications and Extensions ................. 349

15.2.1 Diffusion and Conduction $\ldots \ldots \ldots \ldots \ldots \ldots \ldots \ldots$

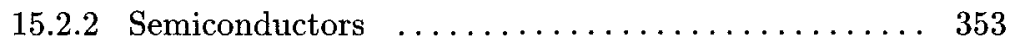

15.2 .3 Metals . . . . . . . . . . . . . . . 357

15.2.4 Thermalization of Electrons in a Conductor ..... 364

15.2.5 Neutron Physics $\ldots \ldots \ldots \ldots \ldots \ldots \ldots \ldots \ldots \ldots$

15.3 The Boltzmann Equation $\ldots \ldots \ldots \ldots \ldots \ldots \ldots \ldots \ldots \ldots$

15.3.1 The Boltzmann Collision Term ........... 371

15.3.2 Densities and Fluxes in a Gas ........... 377

15.3.3 Transport Coefficients of a Gas .......... 381

15.3.4 Gas Mixtures .................. 388

15.3.5 Brownian Motion $\ldots \ldots \ldots \ldots \ldots \ldots \ldots \ldots \ldots \ldots$

15.4 Microscopic Reversibility vs Macroscopic Irreversibility . . 395

15.4.1 The Boltzmann Description ............ 395

15.4.2 The $H$-Theorem $\ldots \ldots \ldots \ldots \ldots \ldots \ldots \ldots \ldots . \ldots \ldots$

15.4.3 Irreversibility of the Boltzmann Equation . . . . . 402

15.4.4 Boltzmann and Thermodynamic Entropies ..... 408

15.4.5 Spin Echoes $\ldots \ldots \ldots \ldots \ldots \ldots \ldots \ldots \ldots \ldots \ldots \ldots 411$

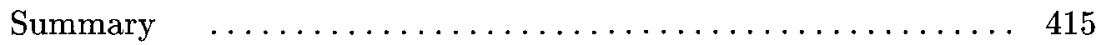

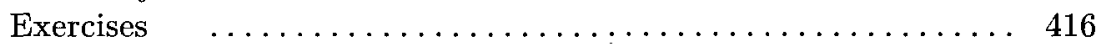

15a Diffusion in Solids; Doping $\ldots \ldots \ldots \ldots \ldots \ldots \ldots 416$ 
15b Impedance in the Lorentz Model . . . . . . . . . 417

15c Dynamics of a Lorentz Gas Particle .......... 419

15d Hall Effect .................... 421

15e Absorption, Stimulated and Spontaneous Emission $\ldots \ldots \ldots \ldots \ldots \ldots \ldots 423$

$15 f \quad$ Heat Transfer in Stars . . . . . . . . . . . 425

16. Problems $\ldots \ldots \ldots \ldots \ldots \ldots \ldots \ldots \ldots \ldots \ldots \ldots \ldots \ldots \ldots \ldots \ldots$

16.1 Paramagnetism of Spin Pairs $\ldots \ldots \ldots \ldots \ldots \ldots \ldots \ldots \ldots 44$

16.2 Elasticity of a Polymer Chain $\ldots \ldots \ldots \ldots \ldots \ldots \ldots \ldots \ldots$

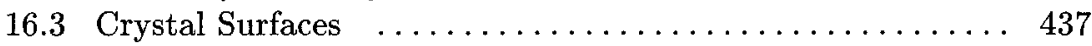

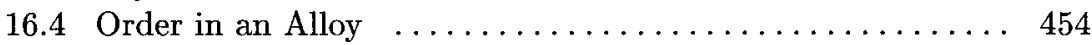

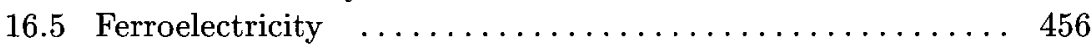

16.6 Rotation of Molecules in a Gas ............... 460

16.7 Isotherms and Phase Transition of a Lattice Gas . . . . . 463

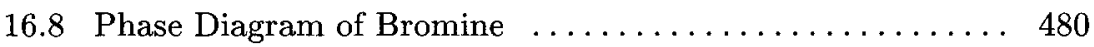

16.9 White Dwarfs $\ldots \ldots \ldots \ldots \ldots \ldots \ldots \ldots \ldots \ldots \ldots \ldots \ldots$

16.10 Crystallization of a Stellar Plasma $\ldots \ldots \ldots \ldots \ldots \ldots \ldots 499$

16.11 Landau Diamagnetism .................. 503

16.12 Electron-Induced Phase Transitions in Crystals . . . . . . 508

16.13 Liquid-Solid Transition in Helium Three . . . . . . . . . 516

16.14 Phonons and Rotons in Liquid Helium ........... 521

16.15 Heat Losses Through Windows . . . . . . . . . . . 539

16.16 Incandescent Lamps . . . . . . . . . . . . . . . 542

16.17 Neutron Physics in Nuclear Reactors . . . . . . . . . 552

16.18 Electron Gas with a Variable Density . . . . . . . . . . 558

16.19 Snoek Effect and Martensitic Steels ............ 563

Conclusion: The Impact of Statistical Physics . . . . . . . . . 571

Subject Index $\ldots \ldots \ldots \ldots \ldots \ldots \ldots \ldots \ldots \ldots \ldots \ldots \ldots \ldots \ldots \ldots$

Units and Physical Constants ................... 604

A Few Useful Formulae $\ldots \ldots \ldots \ldots \ldots \ldots \ldots \ldots \ldots \ldots$ 